Archives de sciences sociales des religions

161 | Janvier-Mars 2013

Messianismes et anthropologie entre France et Italie I Figures et substituts de saints

\title{
Introduction : figures et substituts du saint. La fabrique rituelle
}

Jean-Pierre Albert, Raymond Jamous et Antoinette Molinié

\section{(2) OpenEdition}

Journals

Édition électronique

URL : http://journals.openedition.org/assr/24900

DOI : $10.4000 /$ assr.24900

ISSN : $1777-5825$

Éditeur

Éditions de l'EHESS

Édition imprimée

Date de publication : 1 avril 2013

Pagination : 165-173

ISBN : 13-978-2-7132-2394-5

ISSN : 0335-5985

Référence électronique

Jean-Pierre Albert, Raymond Jamous et Antoinette Molinié, « Introduction : figures et substituts du saint. La fabrique rituelle », Archives de sciences sociales des religions [En ligne], 161 | Janvier-Mars 2013, mis en ligne le 30 mai 2016, consulté le 01 mai 2019. URL : http://journals.openedition.org/ assr/24900 
Figures et substituts de saints 



\section{Jean-Pierre Albert, Raymond Jamous, Antoinette Molinié}

\section{Introduction : \\ figures et substituts du saint. La fabrique rituelle}

Les notions de saint et de sainteté seront adoptées ici à titre heuristique non seulement pour le christianisme mais aussi, par extension, pour parler de personnages et de manifestations à première vue analogues dans l'islam ou d'autres contextes religieux. Nous nous intéresserons seulement à des cultes déjà constitués, qui s'adressent à des morts et/ou des esprits, des divinités locales, bref des êtres "surnaturels ». Cela revient à laisser de côté un autre aspect de la « fabrique des saints ", l'étude des formes sociales de la reconnaissance de virtuoses religieux crédités de pouvoirs surhumains. Comment, et à quelles conditions, des notions qui constituent des catégories internes au monde chrétien peuvent-elles devenir un outil conceptuel dans le cadre d'une approche comparatiste ? Nous partons de l'hypothèse que l'inadéquation de la traduction de certains termes d'une langue par "saint » et «sainteté » nous permet de revoir les significations données à ces notions, de les affiner et de les préciser. Ce sont les difficultés même de l'usage de ces termes de "saint " et de "sainteté " qui sont bonnes à penser dans une perspective comparative.

Au-delà de l'exégèse religieuse (notamment chrétienne) qui définit les formes de la sainteté et nous situe dans un point de vue interne à une religion, la perspective anthropologique que nous avons choisie considère les différents contextes sociaux dans lesquels sont construites des entités surnaturelles supposées interagir avec leurs producteurs. Dans certaines traditions, notamment celles du christianisme et de l'islam, ces entités, en l'occurrence les saints, ont des points communs avec la divinité ou des êtres comme les démons ou les esprits. Ils s'en démarquent cependant par leur humanité. Mais ils se distinguent aussi des humains ordinaires par leur conduite durant leur vie et, au-delà de la mort, par leur présence et leur action en faveur ou au détriment des humains. S'ils ne sont pas des vivants comme les autres, ils ne sont pas non plus des morts comme les autres : ils n'ont pas pour vocation de devenir des ancêtres, ils ne sont pas non plus de mauvais morts qui reviennent hanter les vivants.

Il est vrai que d'autres traditions insistent sur la continuité entre les dieux, les saints et les morts ou proposent des conceptualisations différentes de la chaîne 
des êtres. Il s'agit évidemment de comprendre le contenu que chaque culture ou chaque religion donne à ces trois catégories. Cependant il faut noter qu'elles ont en commun un statut ontologique particulier, celui du "surnaturel » dans le langage de notre tradition culturelle. Cette notion, on le sait, est en général l'objet d'un réel discrédit dans le domaine de l'anthropologie du religieux. Auguste Comte déjà notait son lien avec la culture européenne en soulignant qu'elle ne peut exister que là où l'on dispose d'un concept de la nature, conçue comme ensemble des phénomènes régis par des lois déterministes immanentes. Cette critique (reprise par Émile Durkheim et sous une forme peu différente par Jean Pouillon) est en fait elle-même discutable ${ }^{1}$, entre autres raisons parce qu'elle ne signifie nullement que la seule alternative à la dichotomie nature/surnaturel soit la représentation d'un monde où tous les êtres auraient le même statut ontologique ou entreraient dans un discours homogène (du point de vue de son mode de construction, des procédures de validation, etc.). On peut au contraire penser que les représentations religieuses intègrent toujours des énoncés contre-intuitifs et sont localement reconnues comme distinctes des savoirs empiriques sur la nature. Ainsi les dieux, les saints et les morts sont-ils crédités de pouvoirs ou de propriétés (ubiquité, capacité d'action à distance, immortalité, etc.) en contradiction avec les représentations usuelles de la personne, de l'être vivant, de la causalité. Prendre en compte cette "règle de construction " probablement universelle en son principe, mais susceptible de réalisations variées, permet de produire des définitions de statuts ontologiques ou de rôles échappant au soupçon de « christianocentrisme».

Un second point commun aux phénomènes qui nous intéressent peut être noté. Les entités surnaturelles ne sont pas immédiatement données dans l'expérience des hommes (sauf "expériences du surnaturel » souvent réservées à un nombre limité de virtuoses religieux). Aussi les religions recourent-elles de façon générale à des objets sensibles destinés à les évoquer : images, reliques, etc., que nous réunissons ici sous le nom de "substituts ". C'est là une constatation facile à faire : y compris les traditions religieuses les plus iconophobes et les plus spiritualistes ne peuvent se passer de symboles ou d'objets sacrés impliqués dans le culte. Mais il faut aussi ajouter (et c'est là le cœur de notre problématique) que le lien entre le substitut et l'entité de référence n'est pas donné lui non plus, il n'existe ou ne devient agissant que lorsqu'il est produit, sur le mode de l'événementialité, à travers une situation généralement rituelle. Autrement dit, l'évocation de l'entité surnaturelle ne devient prégnante que lorsqu'elle est portée par un contexte, étayée par une sorte de pédagogie qui s’ancre dans les pratiques.

1. Voir Jean-Pierre Albert, "Le surnaturel : un concept pour les sciences sociales?", postface au numéro thématique des Archives de sciences sociales des religions " Des expériences du surnaturel ", nº 145, 2009, p. 147-159. 


\section{Le traitement rituel de la sainteté}

Nous faisons donc l'hypothèse que les entités postulées par les religions sont dans une large mesure le produit des pratiques et, au premier chef, des rituels. Si l'accomplissement de ceux-ci présuppose une croyance dans l'esprit des acteurs, il faut encore se demander en quoi la pratique elle-même institue (ou reconstitue) la crédibilité de ces représentations préalables. Par ailleurs, nous l'avons dit, la relation avec les saints passe en général par l'intermédiaire d'objets rituels : reliques, lieux, traces écrites, tombes, images ou même êtres humains qui portent les stigmates de leur saint. Les objets peuvent être plus ou moins élaborés et plus ou moins détachés de leur référent, les pouvoirs qui leur sont reconnus sont également variables. Mais ils ont en commun de donner lieu à des processus d'appropriation par les particuliers ou par des communautés et surtout d'être utilisés pour rendre présentes les entités saintes dans certaines circonstances cérémonielles. Aussi, occupant la place du saint, ses substituts peuvent devenir euxmêmes le centre du culte, l'action rituelle établissant par leur intermédiaire une relation entre le saint et les dévots qui le sollicitent. Dans tous les cas de figure, un processus rituel crée en quelque sorte une chaîne de continuité et de discontinuité entre des êtres humains et les êtres surhumains ou non humains, entre les vivants et les morts, etc. C'est cette chaîne que nous voulons analyser dans différents contextes culturels à travers la notion de substitut.

Cette notion donne à penser que l'objet ainsi désigné a une valeur moindre que celui dont il occupe la place, et qu'il est utilisé faute de mieux. En même temps, si l'entité visée est de l'ordre du surnaturel, sa présentification à travers une réalité de ce monde, donc ontologiquement inférieure, est inévitable. L'attitude des fidèles par rapport à ce type d'objets de culte (reliques corporelles ou périphériques des saints, images, empreintes...) devrait donc être partagée entre la valorisation de l'élément matériel qui, en vérité, se donne dans l'expérience comme seul accessible et, d'un autre côté, la conscience de son imperfection. Cette contrainte se retrouve dans la théologie chrétienne de l'image ou, plus largement, des supports sensibles de la dévotion. En principe, ceux-ci sont destinés à orienter la pensée vers le spirituel, ils ne sont que des intermédiaires sans valeur intrinsèque. Mais on sait bien que ce processus peut échouer et se refermer sur une attitude « idolâtre ».

La question du statut des images dans les diverses confessions chrétiennes a fait l'objet d'une très abondante littérature centrée avant tout sur les figurations (statues ou icônes) et quelques formes d'évocation non figuratives que leur comparaison avec les données iconiques rend particulièrement intéressantes : il semble bien que, dans l'image elle-même, la relation analogique (ou figurative) compte moins que la reconnaissance de la présence de l'être évoqué. Notre apport se veut un approfondissement de cette relation, dans ce qu'elle a d'opaque. D'où la seconde question mentionnée plus haut : à quelles conditions l'évocation devient-elle substantielle ? À ce propos également, on n'est pas en domaine 
inconnu, le problème posé étant celui de l'efficacité (ou l'agentivité) des objets impliqués dans les cultes, qui peut avoir des origines très diverses. En insistant sur l'importance des contextes rituels, nous prenons le risque de déplacer la question - de l'efficacité des substituts à celle du rite en général. Mais c'est aussi une occasion de se demander comment un objet devient rituel, ou encore de repérer des caractéristiques de l'objet qui facilitent son usage ritualisé, par exemple sa réalité de figuration (à concevoir peut-être comme évocation excluant une partie des aspects fonctionnels de l'entité de référence). Il faut sans doute également suivre les intuitions de Jack Goody sur la peur des représentations ${ }^{2}$ : qu'un artefact devienne l'équivalent d'une réalité naturelle suffit peut-être à induire un trouble catégoriel. C'est enfin et surtout une occasion de poser à notre tour un principe plus souvent admis que vraiment suivi : que les objets religieux (tout l'attirail du sacré) n'existent en tant que tels qu'en situation - en situation rituelle. Une fois le temple refermé, la statue cesse de présentifier le dieu ; finie la cérémonie du 11-novembre, on peut sans susciter le scandale appuyer son vélo contre la grille du monument au mort.

Enfin et surtout, ce que montrent les différents exemples analysés ici, c'est la complexité des relations que l'on découvre entre signes et référents, signes de signes, signes devenus eux-mêmes des référents... La pratique du substitut apparaît ainsi comme une stratégie (volontaire ou involontaire de la part des promoteurs des rituels) du détour ou du labyrinthe, une manière de faire perdre leurs repères aux acteurs engagés dans les situations. C'est ce que montrent avec une évidence particulière les incertitudes des fidèles confrontés à des " originaux " et des « copies » d'icônes ou de statues : jusqu'à quel point la reproduction est-elle compatible avec le transfert d'une puissance du modèle à la copie ? Un croyant peut-il admettre avec quelque vraisemblance qu'une image participe plus qu'une autre de l'essence de son modèle ? Il n'est assurément pas indifférent que la Vierge ait été peinte, veut-on croire, par saint Luc, ainsi devenu le pourvoyeur du prototype de son portrait le plus authentique : il tient ainsi un rôle de témoin dans le registre de l'image, et non plus dans celui du texte que lui conférait déjà son statut d'évangéliste. Mais faut-il pour autant isoler une lignée de copies (et de copies de copies) qui seraient plus sacrées que d'autres? Sous quelles conditions (sainteté ou pureté du peintre, par exemple) cette filiation sera-t-elle valide?

Les questions posées à propos des images peuvent sans peine être élargies à la catégorie plus large des substituts. Entrent ainsi dans cette rubrique des opérations telles que le sacrifice, dans lequel les victimes sont considérées comme des substituts du sacrifiant avec des chaînes de continuité et de discontinuité entre différentes catégories d'êtres. Il est significatif dans ce cas que la victime soit le substitut du sacrifiant et non de l'être puissant qu'est le saint. Ce substitut sacrificiel disparaît, alors que l'objet rituel, quant à lui, est durable et peut être réutilisé.

2. Jack Goody, La peur des représentations, Éds. La Découverte, 2003. 
Cependant on peut se demander s'il n'existe pas de rituels sacrificiels liés aux saints dans lesquels les offrandes sont faites à des objets représentant les saints. Dans ces circonstances la manipulation de différents objets se substituant à différents acteurs présents ou absents ont des statuts qu'il est bon de comparer.

Nous pensons donc qu'il faut déplacer le problème de la sainteté à celui du traitement rituel de la sainteté. Si ce changement de perspective s'avère significatif, alors la recherche doit se centrer sur les différents acteurs des rites et leurs positions (officiants, dévots, disciples) ainsi que sur les objets que ceux-ci utilisent pour fabriquer ou refabriquer ce que nous avons convenu d'appeler des saints. Il convient donc de définir les opérations cognitives à l'œuvre, telles la substitution, l'identification, l'équivalence, le remplacement, l'asymétrie, la métaphore, la métonymie, l'analogie, et, bien entendu, les combinaisons entre ces divers procédés.

Toutes ces opérations se déroulent dans des contextes spatiaux et temporels particuliers. À cet égard le calendrier rituel apparaît comme un cadre essentiel : l'objet rituel se transfigure en saint à des moments précis de l'année. Mais il ne faut pas ignorer les circonstances exceptionnelles qui peuvent favoriser l'apparition du saint ou ses manifestations. Par ailleurs différentes catégories de l'espace sont ici pertinentes, du monde socialisé à celui de la marge (forêt, désert, etc.) qui semble particulièrement propice aux êtres infernaux, aux esprits mais aussi parfois aux saints, en passant par le monde cosmique et enfin l'au-delà du monde. Plutôt que d'être enfermés dans un espace particulier, les saints apparaissent souvent comme des personnages qui permettent de relier les différents espaces et les différentes temporalités. Plus encore, ils sont souvent associés aux frontières territoriales et/ou temporelles, qu'ils marquent de leur présence, les transcendant par leur action propre ou par celle de leurs disciples et dévots. Le traitement rituel de la sainteté conduit à analyser ces lieux et ces temps frontières, ces rapports entre centre et périphérie par l'intermédiaire desquels les interactions entre ces personnages « exceptionnels » et les communautés particulières se réalisent.

\section{Une approche comparative des substituts de saints}

Il apparaît évident que c'est par l'ethnographie comparée que l'on pourra éclairer la question de la sainteté à travers l'étude des manipulations rituelles de substituts engagées dans les cultes. Pour chacun des exemples retenus, il convenait donc de distinguer l'approche anthropologique de la théologie. En effet les saints et les pratiques qui les entourent apparaissent bien souvent en dehors de l'orthodoxie, qui parfois les combat. Quelques directions de recherche se dégagent de nos ethnographies.

Les contextes monothéistes, la présence d'un clergé ou d'un culte " officiel » entretiennent un rapport complexe avec les substituts de saints. Comme le note Jean-Pierre Albert dans sa contribution, la religion catholique par exemple ne 
les rejette pas totalement mais distingue, de fait sinon de droit, les « bons substituts » de ceux qui sont contraires aux normes. On retrouve cette même attitude dans le monde musulman : en l'absence d'une Église, ce sont les théologiens et les tenants du dogme qui tolèrent ou rejettent toutes ou certaines formes de culte de saints autour des mausolées. Quoi qu'il en soit, on observe une tension permanente entre, d'une part, l'orientation vers l'au-delà, la médiation avec le ciel privilégiée par une Église ou par un dogme et, d'autre part, des demandes concernant le monde ici-bas qui, dans le christianisme comme dans l'islam, sont adressées aux saints dans les contextes de pratique dont ils sont le centre : fêtes votives, pèlerinages, etc. Ce décentrement des attentes religieuses permet ainsi de comprendre que les substituts acquièrent eux-mêmes une place centrale, dans une perspective qui peut être désignée de façon normative comme magique ou superstitieuse.

On peut se demander si le procédé même de substitution est compatible avec le monothéisme : comment fabriquer le substitut d'un dieu unique ? Emma Aubin-Boltanski traite des relations difficiles entre l'islam et le culte des saints. À partir de l'exemple du sanctuaire de Nabî Mûsâ en Cisjordanie, elle explore l'incertitude qui entoure la tombe d'un saint en islam. Celle-ci marque à la fois la présence d'un être vivant, intercesseur efficace entre les hommes et Dieu et l'absence d'un personnage du passé, mort depuis de nombreux siècles. L'incertitude sur la présence/absence de l'être exceptionnel renvoie à une longue série de doutes et de questionnements sur le rôle d'intermédiaire des saints, la licéité du culte qui leur est rendu et leur efficacité. Ces doutes et les basculements qu'ils génèrent sont envisagés comme un élément central du rapport à Dieu qui se construit autour des tombeaux de saints. Les fidèles musulmans oscillent à l'intérieur d'un spectre d'attitudes communautaires à l'égard de la sainteté dont les deux pôles sont, d'un côté, une attitude de rejet qui consiste à rappeler la transcendance absolue de Dieu et, d'un autre côté, la reconnaissance de l'existence, parmi les hommes et en des lieux d'élection, d'une présence divine.

Il n'est pas rare en effet que les rituels de fabrication des saints bouleversent, inversent ou même transgressent les règles du culte religieux. Les substituts des saints constituent des observatoires privilégiés des polythéismes dissimulés derrière les monothéismes. C'est ainsi qu'Antoinette Molinié décrit comment, durant le pèlerinage de la Vierge del Rocio, en Andalousie, les jeunes gens se livrent au " rapt de l'image de la Vierge " après la messe : ils escaladent la grille du chœur, bousculent le curé, s'emparent du trône en argent de la Vierge et se ruent avec la statue vers la sortie, dans une incroyable bousculade, sans intervention aucune des hommes d'Église. Pourtant ces manipulations violentes et peu orthodoxes de l'image ont pour effet de l'établir dans un statut de Reine concernant directement le champ du politique.

Raymond Jamous étudie au Maroc des rites confrériques de possession comprenant, à première vue, des actes interdits par la loi religieuse : les adeptes 
boivent le sang de l'animal sacrifié alors qu'il faudrait le laisser couler, mangent ses entrailles crues alors qu'elles sont interdites à la consommation. La manipulation des substituts permet d'obtenir le renouveau de la société confrérique et son implantation territoriale, ainsi que de capter la baraka du saint au bénéfice des adeptes comme des sacrifiants.

Il faut noter le contraste entre, d'une part, les substituts anthropomorphes comme les Vierges espagnoles décrites par M. Albert-Llorca et A. Molinié, et, d'autre part, les représentations plus ou moins abstraites (ou non figuratives) comme la tombe du saint musulman dans l'analyse d'Emma Boltanski, ou encore le sang, véhicule de la baraka du saint, tel que l'analyse R. Jamous. Ce cas marocain montre qu'il existe des formules intermédiaires : des personnes humaines peuvent jouer le rôle de substitut dans des contextes rituels particuliers. C'est ce que montre aussi l'exemple japonais présenté par Laurence Caillet, qui permet de questionner la définition même de l'entité surnaturelle et celle de substitut. Une farce présentée dans un théâtre de marionnettes met en scène un sculpteur qui, faute de pouvoir fabriquer la statue d'une divinité, prend sa place et reçoit les offrandes de dévots qui ignorent cette tromperie. Quand la fausse statue urine, c'est-à-dire qu'un jet de liquide est lancé par le maître des marionnettes (qui est selon certaines traditions le sculpteur reconverti), des femmes se précipitent pour recevoir ce liquide divin fécondant. La farce devient rituel, la fausse statue représentée par une marionnette devient, pour les spectatrices, la divinité, l'urine devient une semence divine. Cela montre comment la présence d'un substitut, et même d'un faux substitut, est manipulée par les spectateurs devenus acteurs rituels.

Dans ces différents exemples le rituel, utilisant des substituts de tout genre, s'éloigne des règles de la vie ordinaire, définit ses propres mécanismes faisant de la transgression, de l'inversion ou encore de la farce un moyen d'agir de la divinité. Il n'est nullement question ici de savoir si ces êtres de l'au-delà ou de la surnature consentent ou ont une intentionnalité. Ils sont là pour donner aux dévots ou aux vivants cette source de vie qui est l'objet du rituel, comme l'a souligné A. M. Hocart dans son ouvrage Rois et courtisans.

Le substitut peut se dédoubler, comme c'est le cas des Vierges du sud de la France et de l'Espagne étudiées par Marlène Albert-Llorca. En pays valencien, l'effigie principale, la « vraie statue » de la Vierge patronne de Valence, la Mare de Déu dels Desamparats qui incarne la divinité, sort rarement du sanctuaire dans les processions et c'est une autre effigie, appelée la Peregrina, qui prend sa place. La ferveur qui accueille cette dernière lors de sa sortie est immense : c'est que la logique du rituel la rend équivalente à la statue principale. Mais cette dévotion à l'égard de la Peregrina cesse lorsque la «vraie statue » redevient accessible dans le sanctuaire. On retiendra aussi que le mouvement apparaît comme un facteur essentiel de l'efficacité représentative du substitut. 
On voit bien que, parfois, deux ou plusieurs substituts interviennent dans les rituels. Dans les exemples cités par M. Albert-Llorca, c'est le dédoublement du substitut qui opère. A. Molinié fournit, toujours pour l'Espagne, un exemple où une même image va prendre différentes formes. La statue de la Vierge del Rocío apparaît comme une Reine mais, à d'autres occasions, comme une bergère, selon les contextes et les moments rituels. Plus encore, elle reçoit dans son sanctuaire la visite et pourrait-on dire l'allégeance de nombreuses confréries conduisant des substituts de la Vierge matérialisés par des étendards. Cette divinité trace ainsi son " espace de grâce ", comme le souligne bien A. Molinié, et l'on voit se dessiner des hiérarchies rituelles entre substituts : en premier lieu une statue et son double, mais aussi de multiples substituts classés par rangs d'ancienneté.

Il est vrai que les substituts des saints se prêtent souvent à des manipulations politiques.

Valérie Robin montre bien comment, dans l'imaginaire et la mémoire d'un village andin, saint Louis devient le protecteur de la communauté en proie aux violences de la guerre civile. Selon la légende, ce saint d'origine française planta son bâton au milieu d'un lac de la cordillère andine. Le lac s'assécha tandis qu'un cèdre de grande taille se mit à pousser, rappelant évidemment le chêne d'un saint Louis plus orthodoxe. Cet arbre est identifié comme "le bâton de saint Louis le Neuvième ", le saint patron du village. V. Robin rapporte comment, dans le souvenir des villageois, saint Louis les protégea contre la violence des militaires et des maoïstes du Sentier lumineux. C'est autour de l'arbre, devenu substitut du saint patron, que les villageois organisent leur défense. Ce substitut végétal se constitue ainsi en mémoire de la communauté, débordant l'action rituelle pour devenir un enjeu politique.

Cette fonction politique et mémorielle est bien analysée par Michèle Baussant à travers le culte de la Vierge de Santa Cruz d'Oran. En effet ses répliques constituent, avec leur environnement rituel, une ressource identificatoire et une forme de patrimoine partagé pour des populations exilées. Le travail de M. Baussant met ainsi en évidence la place des différents acteurs en présence et la manière dont ils s'auto-définissent à travers leur rapport à la Vierge, à sa statue et à l'espace que ses temps d'immobilité, de déplacements et ses dédoublements contribuent à spécifier. Dans le même temps, on saisit comment le substitut fait aussi fonction de trace ou de relique et comment, à travers lui et les temps rituels auxquels il participe, s'articulent différents espaces et rapports au temps, permettant de transformer une expérience commune, l'exil, en "propriété permanente ».

Finalement ces différentes formules de fabrication de substituts des saints permettent d'éclairer la notion même de sainteté. En partant du champ restreint de l'usage de ce terme dans le monde du christianisme, nous sommes parvenus à entrevoir un concept utile à l'anthropologie du religieux dans d'autres contextes. 
En même temps, sans spéculations mais avec les outils indispensables de l'ethnographie et de la comparaison, nous espérons avoir proposé quelques pistes de recherche sur des thèmes classiques de cette discipline, à la fois distincts et complémentaires des objets habituels de la sociologie des religions.

Jean-Pierre ALBERT

Laboratoire interdisciplinaire solidarités sociétés territoires,

Centre d'anthropologie sociale

(UMR 5193, EHESS, CNRS, Université de Toulouse)

albert@ehess.fr

Raymond JAMOUS

Laboratoire d'ethnologie et de sociologie comparative,

Université Paris Ouest-Nanterre-La Défense raymond.jamous@sfr.fr

Antoinette MOLINIÉ

Laboratoire d'ethnologie et de sociologie comparative - CNRS

Université de Paris Ouest-Nanterre-La Défense antoinette.molinie@mae.u-paris10.fr 
